\title{
Vinculación entre usuarios, biblioteca y núcleos académicos en el uso de los recursos de información: una propuesta de alfabetismo informacional en la UNAM
}

\author{
Interaction of users, library and academic staff nuclei in the use of information resources:
} an informational literacy proposal at UNAM

\begin{abstract}
Rosa Guadalupe Valadez Olguin (1), Jessica PÁez Arancibia (2), Margarita Zapata Guerrero (3), María Esther MonRoY BALdi (4), Isabel ORTEGA MONDRAGón (5), Arturo SÁnCHEZ MondRAGón (6), Filemón MondRAgón (1), Gloria Adriana HeRnÁNDEZ SÁNCHEZ (8) y Orlanda Angélica GARRIDo YÁñes (9)

Facultad de Estudios Superiores Cuautitlán, Universidad Nacional Autónoma de México, Cuautitlán Izcalli 54700, México, (1) fesc@servidor.unam.mx. (2) jessica@servidor.unam.mx.

(3) margaret@servidor.unam.mx. (4) mesther@servidor.unam.mx. (5) isaortegam@yahoo.com.mx. (6) asmondragon_unam@yahoo.com.mx. (8) adria568@hotmail.com. (9) orlanda@dgb.unam.mx.
\end{abstract}

\section{Resumen}

Se analiza el rol de la biblioteca como prestadora de servicios de información de calidad que coadyuve en las funciones sustantivas de la universidad, y del compromiso que ésta tiene con la sociedad, en formar profesionistas sólidamente preparados en sus respectivas áreas de conocimiento y como poseedores de la información, que les permita una constante actualización en sus campos de conocimiento y en su entorno laboral. Los recursos y habilidades en la búsqueda y recuperación de información son herramientas que no debemos soslayar en la formación de nuestros educandos, para lograr una educación de altura en el contexto informacional y en las nuevas tecnologías de información, así como en el continuo proceso de la investigación científica que todo país requiere para su propio desarrollo. En este entorno la biblioteca debe constituirse como un bastión fundamental para este proceso de formación que haga posible a la Universidad cumplir sus fines de docencia, investigación y extensión de la cultura. La propuesta de alfabetismo informacional incluye: formato de solicitud y entrevista con docentes de cursos presenciales, manual interactivo para el desarrollo de competencias en el uso de los recursos digitales de la institución y curso en línea con las mismas temáticas.

Palabras clave: Bibliotecas universitarias. Alfabetismo informacional. Vinculación academia-biblioteca. Vinculación biblioteca-usuario. Recursos de información.

\section{Marco de referencia}

Las Facultad de Estudios Superiores Cuautitlán es una dependencia académica de la Universidad Nacional Autónoma de México, ubicada a $70 \mathrm{~km}$. del campus central con los enormes

\begin{abstract}
In this paper we analize the role of the university library as a provider of quality information services that collaborate with the basic functions of the University and its commitment with society: teaching well prepared professionals in vast areas of knowledge who possess the capacity of acquiring all the new information that is produced in their field and in their working environment. Skills and abilities in the search and retrieval of information is a must in the formation of our students, if we want them to be immersed, not only in the informational context and in the new methodologies of information, but also in the steady process of scientific research that a good university education implies, process that any country needs for getting a sustainable development. Our proposal of informational literacy includes required application forms, interviews with professors and instructors of classroom courses, an interactive manual for the development of competence in the use of digital resources the university offers, and one on line course presenting the manual.
\end{abstract}

Keywords: University libraries. Online teaching. Informational literacy. Information resources. Teachinglearning process. 
a la creciente demanda estudiantil para acceder a los programas académicos de la facultad, nos ha permitido como Sistema Bibliotecario integrarnos a los esquemas de crecimiento en recursos de información impresa y digital en los que está inmersa nuestra Institución.

En la actualidad la Institución cuenta con los siguientes recursos: 6 millones de volúmenes, 13,500 suscripciones a revistas científicas (impresas y distribuidas en las diversas bibliotecas); y, en formato digital, 320,000 tesis impresas, 10.071 libros electrónicos, 248 bases de datos, 345 materiales de consulta, 73,500. tesis digitales y 56,504 revistas electrónicas.

Este segmento académico de la UNAM tiene una oferta educativa altamente multidisciplinaria que comprende 13 licenciaturas, 7 maestrías y 5 doctorados. Las áreas del conocimiento que proveen esta oferta educativa está comprendida en las siguientes áreas: Ciencias Biológicas y de la Salud, Ciencias Fisico-matemáticas, Ciencias Sociales, y Humanidades. La comunidad académica está compuesta por aproximadamente 13,000 alumnos y 1,300 docentes.

Con el advenimiento de Internet y el uso desmedido de buscadores y metabuscadores, constatamos que el uso de los recursos de información digital e impresos suscritos y adquiridos por la Institución eran subutilizados. Estos recursos de información se adquieren de manera colegiada por cada una de las dependencias, y de forma consensuada cuando se suscriben y adquieren para toda la Institución. Se busca fundamentalmente que reúnan todas las características académicas y científicas que la Universidad demanda, es decir: que tengan un comité científico, comité de pares, con un buen nivel en cuanto a factor de impacto, que cubra las necesidades académicas y de investigación de la institución, que estos recursos sean utilizados por varias dependencias académicas, y que tengan un uso universal por parte de la comunidad académica.

Pese a los factores antes mencionados la comunidad académica, concretamente los educandos, preferían utilizar los buscadores y metabuscadores que la Internet comercial ofrece, subutilizando los recursos de información adquiridos por la Institución. Este factor nos obligó a vincularnos estrechamente con la comunidad académica-profesores a fin de conocer estrechamente sus necesidades de información concretas y específicas, y de ofrecer y demostrar que la institución poseía los recursos de información necesarios para cubrir estas necesidades. Cabe señalar que, en diversas reuniones y juntas de trabajo, se constató que los núcleos académicos desconocían la cantidad y diversidad de fuentes de información que poseía la institución.

\section{Marco teórico}

Para contextualizar nuestro objeto de estudio, dirigimos nuestros esfuerzos hacia la vinculación estrecha entre los núcleos académicos y de investigación y el sistema bibliotecario de nuestra dependencia. Para ello revisamos algunas definiciones sobre la vinculación. Al analizar el término en diversos diccionarios, constatamos que éste nos llevaba a tres acepciones vinculación, extensión y difusión-, y que las tres se enmarcaban perfectamente en las necesidades que tenía nuestra comunidad académica y en general nuestra institución:

- Vinculación. Según el Diccionario de la Real Academia Española, es la acción y el efecto de vincularse, es decir, la acción de atar o fundar una cosa en otra.

- Extensión. Según el mismo diccionario y el Diccionario Básico del Español de México, es la acción o efecto de extenderse, es decir, de ampliar, diseminar una cosa ampliando su espectro de acción, o, si se quiere, dar mayor amplitud a algo.

- Difusión. De acuerdo a los mismos diccionarios, es la acción de difundirse, es decir extender, esparcir y propagar o divulgar noticias, expresiones de cualquier tipo, conocimientos, actitudes, costumbres etc.

Aunque en nuestra institución la vinculación se enfoca primordialmente desde la relación que existe entre ésta con la sociedad, decidimos adoptar este término para la acción de establecer una acción en otra. En este sentido vertical del término, lo entendemos como la acción de transferir y cimentar un conocimiento en base a uno ya existente. Es decir, los conocimientos disciplinarios de cada una de las áreas que conforman nuestro campus universitarios se verán fortalecidos por la transferencia de conocimientos que nuestro sistema bibliotecario hará de todos y cada uno de los recursos de información que posee la institución; y, viceversa, la biblioteca permeará los contenidos temáticos, subtemáticos y específicos de éstos para fortalecer su acervo impreso, digital y en las futuras tecnologías que aparezcan para el uso y acceso de la información. En este contexto, cabe resaltar la función que nuestro sistema tiene con su comunidad académica de ser facilitadora de las nuevas tecnologías $y$, por ende, del alfabetismo digital. 
En lo inherente a la extensión, nuestro sistema bibliotecario se enfocó a hacer extensivo el conocimiento para uso y acceso a la información a las dependencias de la misma institución que así lo solicitaban, así como a escuelas aledañas a nuestro entorno geográfico.

En cuanto a la difusión, la propuesta se inclinó sobre diversos mecanismos: a) marketing one to one; b) trípticos de divulgación de los recursos de información; c) participación en ferias, semanas, seminarios y simposiums académicos y estudiantiles, dando a conocer los diferentes recursos; y d) presentaciones de proveedores, ferias del libro y eventos generales.

\section{Metodología}

Para tener un diagnóstico de la situación, realizamos un proceso de evaluación en la comunidad académica y estudiantil aplicando un instrumento de medición que nos permitió determinar el alfabetismo informático, el alfabetismo informacional y las necesidades de información que requerían nuestros usuarios conforme al avance de las nuevas tecnologías. Esto se fundamentó en lo señalado por la ACRL/ALA:

Las destrezas en tecnologías de la información capacitan a un individuo para usar ordenadores, aplicaciones informáticas, bases de datos y otras tecnologías para alcanzar una gran variedad de metas académicas, laborales y personales. Los individuos competentes en el acceso y uso de la información necesariamente tienen que dominar determinadas destrezas tecnológicas.

Dicho instrumento de medición fue aplicado a una población determinada por una formula estadística a 366 usuarios quienes en términos generales reconocieron los siguientes aspectos:

En cuanto a los recursos de información, el instrumento arrojó los siguientes aspectos:

1. Desconocían la cantidad y diversidad de recursos de información digital.

2. Los usuarios reconocían la calidad de las fuentes de información respecto a la información que obtenían de Internet.

3. La dificultad más grande para acceder a éstas era el idioma y la especificidad de los términos para una óptima búsqueda.

4. Pedían que se ampliaran las condiciones de acceso remoto para estas fuentes.

5. Lo más relevante fue que consideraron de gran utilidad y aplicación la información de los recursos que ofrecía la Institución.

6. Solicitaron una amplia difusión de cuanto recurso de información se incorporara.
En cuanto a su alfabetismo informático, el cuestionario arrojó los siguientes datos

1. La gran mayoría posee computadora personal y tiene conocimientos de computación y telecomunicaciones y tiene facilidad de acceso a centros de cómputo, dentro y fuera del campus.

2. Aproximadamente el $70 \%$ se inscribe en cursos extracurriculares de computación para fines de actualización y perfección de conocimientos en paquetes específicos de su área de conocimiento.

3. El $54 \%$ tiene cursos de computación en sus currículos académicas en temáticas específicas de su área.

4. La biblioteca adquiere títulos actualizados en el área de computación y telecomunicaciones pero escasos números de volúmenes.

Los datos arrojados en este rubro nos permitieron analizar que en la facultad se mantiene una carga académica en computación adecuada dentro de las currículos académicos y extra curriculares, por lo que pudimos afirmar que cubríamos el alfabetismo informático indispensable para que los educandos accedieran y absorbieran los conocimientos para el alfabetismo informacional.

De acuerdo a los resultados obtenidos nuestros esfuerzos se enfocaron, de manera inmediata, a ofrecer en los períodos intersemestrales una diversidad de cursos a los profesores por áreas del conocimiento, a fin de que conocieran en detalle la diversidad y la calidad de fuentes de información impresa y digital con las que cuenta la Institución.

Lo anterior nos obligó a entrar en un proceso dinámico de intercambio de saberes con los núcleos académicos, en los que ellos nos referían sus necesidades por áreas del conocimiento universalmente conocidas, para adentrarnos posteriormente a necesidades temáticas, subtemáticas, y aspectos concretos y específicos de investigación.

Para establecer nuestro esquema de vinculación con los núcleos académicos y estudiantiles, nuestro sistema bibliotecario estableció 3 tipos de cursos en dos modalidades: presenciales (la gran mayoría) y en línea (en fase de prueba).

Para la elaboración de los contenidos temáticos y ejercicios, tomamos como referencia el siguiente esquema, propuesto por Patricia Hernández Salazar (Figura 1). 


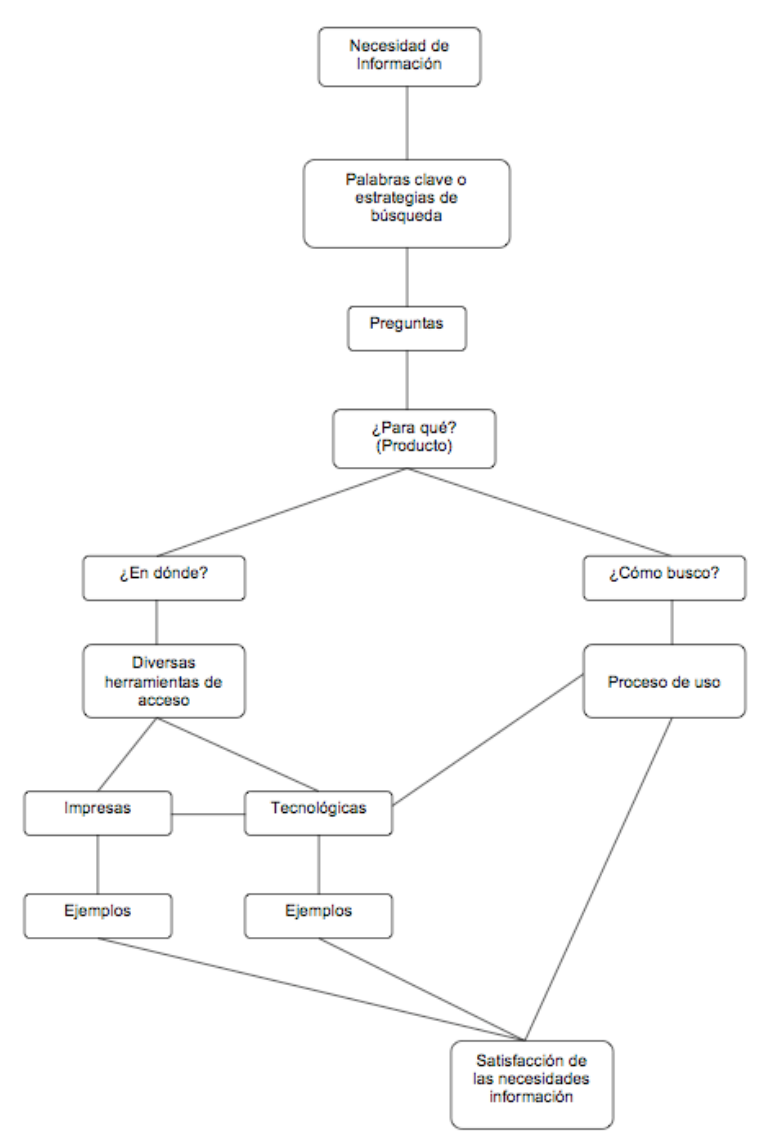

Figura 1. (Salazar, 2004)

Este esquema nos permitió formular los objetivos instruccionales, contenidos temáticos y reorientar dichos contenidos a las temáticas específicas por área del conocimiento

I. Plática de inducción a las fuentes de información digital de la UNAM (dos horas).

1. Plática de inducción a los servicios de las bibliotecas (15 minutos), presentación en Power Point.

2. Demostración a la página electrónica de la FES Cuautitlán (20 minutos).

\subsection{Catálogos electrónicos}
2.1.1 Libros
2.1.2 Tesis
2.1.3 Revistas

3. Demostración de los servicios de la biblioteca digital local (30 minutos).

3.1. Acceso a fuentes secundarias de información:
3.1.1 Diccionarios
3.1.2 Enciclopedias
3.1.3 Clase
3.1.4 Periódica

\subsubsection{Bases de Datos}

II. Curso presencial de introducción a las fuentes de información (cinco horas).

1. Plática de inducción a los servicios de la biblioteca. Presentación en Power Point (20 minutos).

2. Acceso a la página electrónica de la biblioteca de la FESC, Práctica (30 minutos).

2.1. Catálogos electrónicos:
2.1.1 Libros
2.1.2 Tesis
2.1.3 Revistas

3. Acceso a los servicios de la biblioteca digital (40 minutos).

3.1 Acceso a fuentes secundarias de información
3.1.1 Diccionarios
3.1.2 Enciclopedias
3.1.3 Clase
3.1.4 Periódica
3.1.5 Bases de Datos Especializadas

4. Acceso a fuentes de información secundarias, Ejercicios Simples (30 minutos)

\subsection{Revistas digitales}

\subsection{Obtención de documento primario}

III. Curso Taller "Desarrollo de capacidades en el uso y manejo de la información digital"

Dirigido a estudiantes y profesores de la FES Cuautitlán que deseen hacer uso de la información digital de que dispone la biblioteca de esta facultad y de la institución.

Sus objetivos son enseñar a:

- Identificar las fuentes de información digital en las áreas del conocimiento de las disciplinas que se imparten en la Fes Cuautitlán y en general de otras áreas del conocimiento.

- Accesar a las fuentes digitales de información de acuerdo a las necesidades inmediatas de los usuarios.

- Recuperar información digital, ya sea referencial o de texto completo, que le permita al usuarios una identificación directa con sus necesidades de información y la obtención de la misma.

El contenido del programa es el siguiente:

1. Recursos Digitales

1.1. Qué son los recursos digitales de información. 
1.2. Cuáles son los recursos digitales de información.

1.3. Qué recursos digitales se tienen disponibles institucionalmente.

1.4 Qué recursos digitales se disponen de manera local.

\subsection{Otras fuentes de información digital.}

2. Búsqueda de información digital.

2.1. Explicación general de la página digital.

2.2 Acceso a catálogos electrónicos locales: libros, tesis y publicaciones periódicas de la FES Cuautitlán.

2.3. Acceso a fuentes secundarias de información: búsqueda de información en línea por área del conocimiento; arte y comunicación visual, ciencias contable administrativas y sociales, ciencias de la computación e informática, ciencias físico matemáticas e ingeniería, ciencias químico biológicas e ingeniería y ciencias agropecuarias.

2.3.1 Index, bases de datos, enciclopedias digitales, diccionarios digitales.

2.4 Acceso a Fuentes Primarias de Información.

2.4.1 Ciclo y tipos de información.

2.4.2 Estrategia de búsqueda. Detección de necesidades de información. Formulación y estructura de la estrategia, palabras claves, terminología científica por área, manejo de sinónimos y abreviaturas científicas propias del área y cómo localizarlas (palabras claves).

2.4.3 Acceso a libros digitales, revistas digitales, obtención del artículo texto completo.

2.4.4 Recuperación de la información. Impresa, guardado en disco, envío a correo electrónico.

\section{Servicio de alerta}

3.1 Servicio permanente de actualización que ofrecen los recursos digitales de información.

Despliegue de tablas de contenido (toc) en correo electrónico del usuario.

4. Búsqueda de información institucional

4.1. Biblioteca digital de la dirección general de bibliotecas.

4.1.1 Acceso a Librunam, Tesiunam, Seriunam,

4.1.2 Acceso a Libros Electrónicos, Ciencia para Todos.
4.1.3 Acceso a bases de datos por especialidad.

4.1.4 Acceso a revistas electrónicas de texto completo; acceso directo e indirecto.

\section{Acceso a tesis digitales}

5.1 Búsqueda a través de la página de la DGB y de la biblioteca de la FES Cuautitlán; búsqueda por autor, asesor, título; recuperación en formato PDF.

5.2. Acceso a la base de datos Dissertation Abstracts; búsqueda básica, avanzada y recuperación de resumen.

6. Instrucción para la localización de normas y patentes

6.1 Acceso al catálogo de normas nacionales mexicanas; búsqueda temática en la página de la Dirección General de Normas y obtención de documentos primarios.

6.2 Acceso, búsqueda y localización de patentes americanas y europeas; búsquedas temáticas por autor y número internacional de patente.

7. Búsqueda y localización de información en otras bibliotecas digitales

7.1 Acceso a la página del Instituto de Biotecnología de la UNAM.

7.2 Acceso a la página digital de la Biblioteca de DGSCA.

7.3 Acceso a la página digital de la Biblioteca de la Facultad Medicina.

\subsection{Otras Bibliotecas.}

IV. Curso en línea: Desarrollo de las competencias en el manejo de la información de los recursos digitales de la UNAM.

Debido a que no se cuenta con el suficiente personal que imparta estos cursos presenciales al universo potencial de usuarios de información de nuestra comunidad, nuestros esfuerzos se han venido enfocando a desarrollar un recurso en línea con el objetivo de que los usuarios logren:

- Determinar el alcance de la Información requerida identificando el valor y las diferencias entre una gran variedad de recursos y formatos.

- Identificar las fuentes de Información requeridas para satisfacer sus necesidades de información.

- Acceder a ésta con eficacia. 
- Evaluar de manera crítica las fuentes de información, distinguiendo la información comercial, y la información científica y técnica validada por expertos.

- Incorporar la información seleccionada a su propia base de conocimientos, generando nueva información a partir de datos obtenidos de fuentes primarias y secundarias.

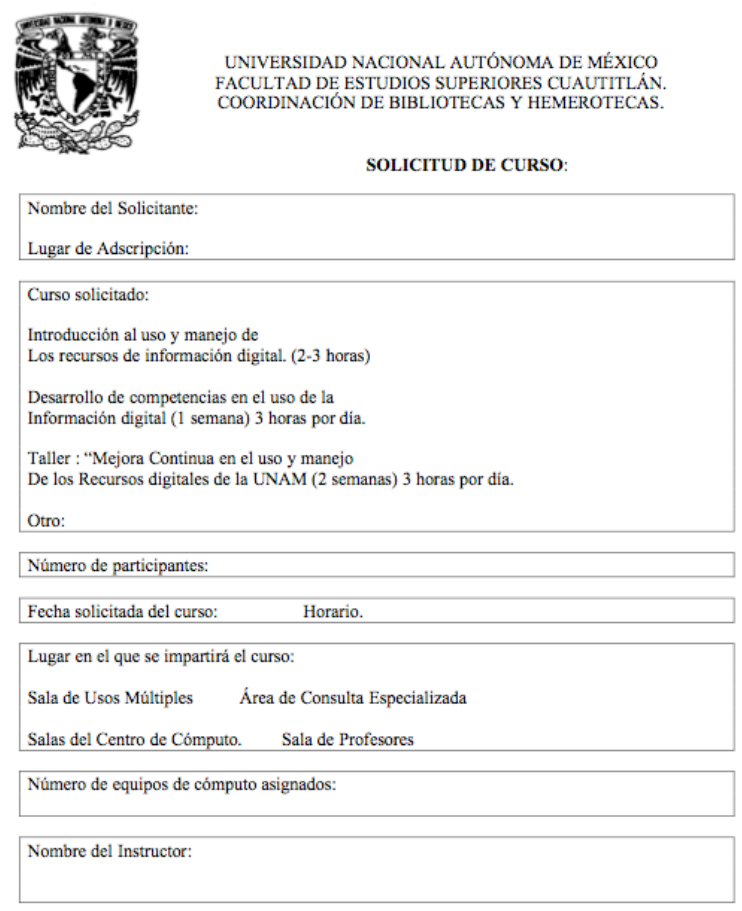

NOMBRE Y FIRMA DEL SOLICITANTE:

FECHA:

Figura 2. Formulario de solicitud de curso

Para consolidar los vínculos con los núclec académicos en cuanto a fortalecer el Alfabetis mo Informacional, planteamos el siguiente e: quema (Figura 4), basado en la experiencia d impartir 56 cursos en las modalidades descrite anteriormente y tomando como base los objet vos establecidos por la Association of Collag and Research Library, en los cuales se señala:

[...] La alfabetización en la información constitu] la base del aprendizaje continuo... Capacita a lc usuarios para dominar los contenidos y ampliar si investigaciones, hacerse más autosuficientes. asumir mayor control de su propio aprendizaje...(') desarrollar un planteamiento metacognitivo c aprendizaje, haciéndoles conscientes de las acciones explícitas que se requieren para reunir, analizar y utilizar la información [...]
- Utilizar la Información de manera eficaz de acuerdo a las tareas académicas y de investigación, aplicando los principios éticos y legales.

Cabe aclarar que el contenido temático será el mismo que hemos descrito anteriormente. En concordancia con los procesos de certificación en los que está inmersa nuestra facultad se establecieron sendos formatos (figuras 2 y 3 ).

\begin{tabular}{l} 
ÁREA GENERAL DE CONOCIMIENTO. \\
CIENCIAS FÍSCIO-MATEMÁTICAS. $\quad$ HUMANIDADES \\
CIENCIAS BIÓLÓGICAS Y DE LA SALUd $\quad$ CIENCIAS SOCIALES. \\
\hline
\end{tabular}

\begin{tabular}{|l|} 
SUB-ÁREA DE CONOCIMIENTO. \\
(Señale la sub-ärea que deseea ser analizada en el curso). \\
CIENCIAS FÍSICO MATEMÁTICAS $\quad$ HUMANIDADES \\
CIENCIAS BIOLÓGICAS Y DE LA SALUD $\quad$ CIENCIAS SOCIALES. \\
\hline
\end{tabular}

\begin{tabular}{|l|}
\hline \multicolumn{1}{|c|}{ TEMÁTICA ESPECÍFICA } \\
(Especifique la(s) temáticas que requiere profundizar. \\
CIENCIAS FÍSICO-MATEMÁTICAS. $\quad$ HUMANIDADES. \\
CIENCIAS BIOLÓGICAS Y DE LA SALUD $\quad$ CIENCIAS SOCIALES. \\
\hline
\end{tabular}

ASPECTOS ESPECífICOS QUE DESEE PROFUNDIZAR EN EL CURSO
a) Estrategia de búsqueda.
b) Acceso a fuentes secundarias de información.
c) Acceso a fuentes primarias de información.
d) Búsqueda y localización de articulos cientificos.
e). Búsqueda y localización de patentes.

Figura 3. Formulario sobre aspectos temáticos

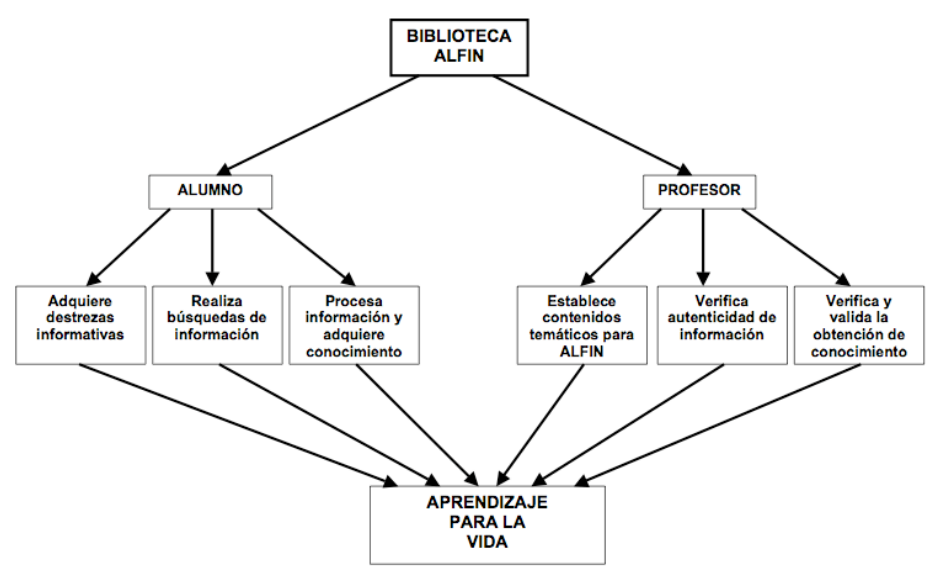

Figura 4. La biblioteca alfabetizadora en información 
De manera adicional fueron diseñados trípticos informativos en los que se describen los servicios digitales de información, que han sido entregados de forma personal a los educandos de las áreas químicos biológicas y humanidades. De manera breve, se les comenta la importancia de la información digital y se les hace hincapié en que estamos en la disposición de que sean atendidos de forma personal y asesorados para su acceso remoto. Cabe señalar que esta acción (Marketing one to one) debe ser extendida a las otras áreas que comprenden nuestra facultad, cuyos trípticos se encuentran en proceso de elaboración. La atención personalizada para promocionar los servicios digitales de información nos ha permitido ser más directos en la divulgación de estos recursos, subsanar dudas inmediatas de los usuarios y promocionar los diversos cursos que ofrece la biblioteca, los cuales, como hemos venido señalando, deben ser sugeridos y propuestos por un profesor.

Cada semestre, se ofrecen los cursos a petición directa y ex profeso de los profesores de 12 asignaturas -6 en el área químico biológica, 4 en el área de ciencias agropecuarias, 2 en el área de humanidades y 1 en el área de ciencias sociales-.

Los enfoques temáticos específicos son proporcionados por los profesores, con base en los contenidos curriculares de sus asignaturas, creando así la necesidad de información para concretar y ampliar el conocimiento de las mismas, tratando de impulsar en el educando el trinomio necesidad-descubrimiento-obtención de la información para generar o concretar el conocimiento.

Falta mucho por hacer $\mathrm{y}$, sobre todo, permear las diferentes áreas del conocimiento que comprende la oferta académica de nuestra facultad. Fuerte trabajo representa el área de ciencias sociales, que históricamente se ha mantenido alejada de las fuentes digitales de información.

\section{Conclusiones}

Un nuevo sistema de comunicación basado en un lenguaje digital universal está integrando globalmente la producción, generación y distribución de la información de nuestras culturas, ofertando sonidos, imagen, texto, video, etc.

Las redes informáticas interactivas - que están creciendo de manera exponencial- crean nuevas formas y canales de comunicación en los distintos entornos: económico, cultural, político y fundamentalmente académico.

Las bibliotecas no han quedado al margen de esta vorágine tecnológica, adquiriendo y propor- cionando a sus comunidades una diversidad de recursos digitales de información; y, además, formando y desarrollando aptitudes, capacidades y habilidades para el uso y acceso de la misma.

En este sentido, el desarrollar metodologías, estrategias y materiales que coadyuven a que los educandos obtengan los conocimientos para aprovechar los recursos de información en sus diversos formatos, es una de las tareas sustantivas a considerar dentro de las bibliotecas. El dominio informacional constituye un nuevo paradigma tecnológico basado en el conocimiento, uso y manejo de las TIC, encaminadas éstas a la transformación del proceso de enseñanzaaprendizaje, a la acumulación de conocimientos y hacia grados complejos en el procesamiento de la información.

La búsqueda del conocimiento es lo que caracteriza la función social y fundamental de nuestras universidades en la formación de los futuros profesionales, y las bibliotecas no han quedado al margen en estos mecanismos de formación, ya que constituyen el apoyo sustantivo de la investigación para la generación de nuevos conocimientos.

Por lo anteriormente expuesto, el implementar programas de alfabetismo informacional debe de ser una actividad prioritaria dentro de los programas de desarrollo de nuestras bibliotecas, permitiendo con ello ser un eslabón entre la información, la academia y la investigación.

En es sentido, la vinculación de las bibliotecas con los núcleos académicos y de investigación es de suma importancia en la transferencia de la información. El desarrollar programas de ALFIN tomando en consideración cuándo y por qué se necesita la información, dónde encontrarla, cómo evaluarla y cómo utilizarla y comunicarla de manera ética debe constituir el objetivo primordial al desarrollar éstos programas, con contenidos temáticos y subtemáticos por disciplina para la concreción del conocimiento.

Las habilidades a promover son, como se ha visto, el reconocimiento de las necesidades de información, qué recursos y en qué formatos se dispone para la obtención de la misma, cómo encontrarla, y cómo gestionar los resultados y explotarlos para la obtención y generación del conocimiento de manera ética y responsable.

Nos resta mucho por hacer, pero la continua evaluación de las experiencias propias y externas nos permitirán avanzar en el proceso de mejora continua de nuestros programas de Alfabetismo Informacional. 


\section{Referencias}

ACRL/ALA. Normas sobre aptitudes para el acceso y uso de la información en la educación superior: Normas de la ACRL/ALA. // Boletin de la Asociación Andaluza de Bibliotecarios. 15:60 (2000) 2. http://www.aab.es/pdfs/ baab60/60a6.pdf

Association of Collage and Research Library (2001). Objetivos de formación para la Alfabetización en información: un modelo de declaración en Bibliotecas Universitarias. ACRL, 2001. 1

Fernández, E. L. (2004). E Learning: Implantación de proyectos de formación on line. México: Alfaomega, 2004.

Hernández-Salazar, P. (2004). Modelo para generar programas sobre la formación en el uso y tecnologías de información. México: UNAM, 2004.

Diccionario de la lengua española - Vigésima segunda edición. http://buscon.rae.es/drael/ (2008-05-28).

Sánchez, J. L. (2006). Introducción al diseño de cursos en línea (Curso en línea impartido en la Facultad de Estudios Superiores Cuautitlan, como parte del PAPIME EN316603, UNAM, 2006).

Valadez, Rosa; [et al.] (2007). Diseño de un curso en línea para la alfabetización informacional bajo el modelo ADDIE: una experiencia en la UNAM. // Ibersid: Revista de sistemas de información y documentación. (2007) 267274.

Valadez, Rosa; [et al.] (2005). La gestión en el uso de los recursos digitales de la información: estudio de casos en la biblioteca de la Facultad de Estudios Superiores Cuautitlán-UNAM. // En las memorias del $7^{\circ}$ Congreso del Capítulo Español de ISKO: La Dimensión Humana de la Organización del Conocimiento. Universidad de Barcelona, España. 1995. 613-624. 